\title{
GMR
}

\section{White mold resistance-associated quantitative trait loci in the Jalo $x$ Small White common bean population}

D.A. Souza ${ }^{1}$, M. Balestre ${ }^{2}$, A.K.A. Pamplona ${ }^{2}$, M.E. Leite $^{1}$, J.A. Dias ${ }^{1}$ and J.B. Santos ${ }^{1}$

${ }^{1}$ Departamento de Biologia, Universidade Federal de Lavras, Lavras, MG, Brasil

${ }^{2}$ Departmento de Ciências Exatas, Universidade Federal de Lavras, Lavras, MG, Brasil

Corresponding author: M. Balestre

E-mail: marciobalestre@dex.ufla.br

Genet. Mol. Res. 15 (3): gmr.15038724

Received April 25, 2016

Accepted June 24, 2016

Published August 26, 2016

DOI http://dx.doi.org/10.4238/gmr.15038724

Copyright $(2016$ The Authors. This is an open-access article distributed under the terms of the Creative Commons Attribution ShareAlike (CC BY-SA) 4.0 License.

ABSTRACT. We aimed to identify simple sequence repeat (SSR)
markers linked to quantitative trait loci (QTLs) associated with white
mold resistance in a segregating population derived from a cross
between common bean cultivars Jalo and Small White, in the Southern
State of Minas Gerais. Parents were crossed to obtain the $\mathrm{F}_{2}$ generation
of 190 plants. From these, $\mathrm{F}_{2: 3}$ and $\mathrm{F}_{2: 4}$ progenies were obtained for
phenotypic evaluation. DNA was extracted from $\mathrm{F}_{2}$ plants and parents
for genotyping with SSR primers. For phenotypic assessment by the
straw test, we used $190 \mathrm{~F}_{2: 3}$ progenies and six lines in a triple-lattice
design of $14 \mathrm{x} 14 \mathrm{~m}$. Nine trials were conducted for phenotyping by
the oxalic acid method to evaluate $177 \mathrm{~F}_{2: 4}$ progenies, in addition to the
two parents and two common treatments represented by the Jalo and

Genetics and Molecular Research 15 (3): gmr.15038724 
Corujinha lines. The experimental design for the oxalic acid method was completely randomized with three replicates. Adjusted means of evaluations related to $\mathrm{F}_{2: 3}$ and $\mathrm{F}_{2: 4}$ were used to identify QTLs by using the moving away method from the marker under Bayesian analysis. The markers GATS91, BM197, and X60000 stood out with high heritability and with effects on disease reduction by the straw test; therefore, these markers are promising for selection. The markers BM183, BM189, and SSR-IAC143 were associated with the QTLs identified by oxalic acid, providing greater effects in white mold resistance with high heritability. Based on the oxalic acid and straw test methods, the most prominent marker was PVBR189.

Key words: Phaseolus vulgaris; Sclerotinia sclerotiorum; Bayesian analysis; Oxalic acid; Straw test

\section{INTRODUCTION}

White mold, caused by the fungus Sclerotinia sclerotiorum (Lib.) De Bary, is one of the most devastating fungal diseases of beans, because it limits yield potential and also reduces the quality of seeds and pods. The occurrence of white mold has increased in recent years, causing large losses in commercial plantations. Outbreaks can cause an average loss of 30\%. However, under favorable climatic conditions, these losses may reach $100 \%$ in susceptible cultivars (Singh and Schwartz, 2010).

Considering the impact of this disease on bean crops, researchers have sought strategies to evaluate resistance to white mold. Among these strategies, fungal mycelium has been used in the straw test (Singh and Téran, 2008), which is a non-destructive method, allowing rapid advances in breeding programs performed to obtain resistant progenies. An indirect method is also used for assessment, in which genotypes are evaluated according to the resistance of the plant to oxalic acid. This method was developed based on evidence that S. sclerotiorum uses oxalic acid to invade bean plants, and acid resistance can function as an indirect mechanism of disease control (Kolkman and Kelly, 2000).

Carvalho et al. (2013) concluded that both the straw test and oxalic acid methods could be used together, because they can select progeny carriers of different white moldresistance mechanisms.

Although the evaluation methods are well established, success in breeding for resistance to white mold has been limited mainly due to lower resistance levels of lines, complex trait inheritance, and high environmental influence, which together reduce heritability (Carneiro et al., 2010). In this sense, the development of breeding strategies to identify superior genotypes in the early stages of breeding programs is of great interest.

An alternative is the adoption of molecular marker-assisted selection (MAS). MAS permits the introduction or overexpression of genes or quantitative trait loci (QTLs) in susceptible genotypes or populations of interest. In this way, some QTLs conferring resistance to white mold, determined by field and greenhouse tests, were identified over the 11 linkage groups in common bean (Ender and Kelly, 2005; Maxwell et al., 2007; Mkwaila, 2013).

Information provided by molecular markers is useful in the selection process only when associated with QTLs that are involved in disease control, mainly in sources of adaptive

Genetics and Molecular Research 15 (3): gmr.15038724 
resistance. Accordingly, the identification of QTLs associated with white mold resistance, as assessed by different methods, permits the identification of more QTLs, as well as validating those already described in the literature.

The objective of this study was to identify simple sequence repeat (SSR) markers linked to QTLs controlling resistance to white mold, by using the straw test and plant reaction to oxalic acid in a segregating population derived from a cross between the cultivars Jalo and Small White.

\section{MATERIAL AND METHODS}

\section{Genetic material}

The lines Jalo EEP 558 and Small White were used as the parents. The cultivar Jalo EEP 558 is semiprostrate, plant type III, with indeterminate growth, grain of intermediate brightness, trade group Manteigão, 100-grain weight of 30-40 g, medium cycle, and adapted to the Southeastern Brazil. It is moderately resistant to white mold as determined by the straw test and oxalic acid. The cultivar Small White is of Mesoamerican origin, has white beans, is plant type IV, has long internodes, and is prostrate. In field trials, it has not shown resistance to white mold. Although the Small White is not adapted to Brazilian conditions, it is one of the few genotypes that are compatible with Jalo, which is of Andean origin.

The parents were crossed to obtain the $\mathrm{F}_{1}, \mathrm{~F}_{2}$ generations, and $\mathrm{F}_{2: 3}$ and $\mathrm{F}_{2: 4}$ progenies, which were used in the experiments.

\section{Progeny genotyping}

DNA from $190 \mathrm{~F}_{2}$ plants and both parents was extracted according to the method described by Pereira et al. (2007). Nucleic acids were rehydrated in Tris/EDTA buffer and quantified on $1 \%$ agarose gel using DNA markers of known concentrations. After quantitation, DNA was diluted to a concentration of approximately $10 \mathrm{ng} / \mu \mathrm{L}$ for use in subsequent reactions.

First, polymorphisms were investigated in the parents with 600 pairs of random microsatellite primers (SSR) of Phaseolus vulgaris, whose sequences are available in the Bean Improvement Cooperative (BIC, 2007). After checking the polymorphism in the parents, 70 primers considered polymorphic were used to genotype the $F_{2}$ population of 190 plants. Genotypes of the SSR markers were identified with scores of $-1,0$, and 1 for homozygous with fewer base pairs, heterozygous, and homozygous with a greater number of base pairs, respectively. For all markers, the DNA fragment (allele) from the parent Jalo was considered as $\mathrm{A}^{1}$ and the allele from the parent Small White was considered as $\mathrm{A}^{2}$. At each locus, changes in allele frequencies were checked using the $\chi^{2}$ test, which determines whether the frequencies deviate casually from those expected. The general expression is represented by

$$
\chi^{2}=\sum_{i} \frac{\left(F O_{i}-F E_{i}\right)^{2}}{F E_{i}}
$$

in which $F O_{i}$ is the frequency observed in class $i$ and $F E_{i}$ is the expected frequency of class $i$. The segregation hypothesis was 1:2:1 in $\mathrm{F}_{2}$, and loci with significance at 5\% probability or less were considered as having segregation distortion.

Genetics and Molecular Research 15 (3): gmr.15038724 


\section{Evaluation of resistance to white mold using the straw test}

Phenotypic evaluation was performed in the field using $\mathrm{F}_{2: 3}$ progenies during the winter growing season. The experimental design used was a $14 \times 14-\mathrm{m}$ triple lattice, the 190 progenies were considered as treatments, and both parent lines (Jalo and Small White), the lines Cornell 605, G-122, and CNFC 9506, which have partial resistance to white mold, and the cultivar Corujinha, which is susceptible to disease, were considered as controls. The experimental plot consisted of a $1-\mathrm{m}$ row with $0.60-\mathrm{m}$ spacing between rows and a sowing density of 15 seeds per meter.

We used an aggressive isolate of $S$. sclerotiorum identified by Silva et al. (2014). We obtained the inoculum of disinfected sclerotia in potato-dextrose-agar medium at $20^{\circ} \pm$ $3^{\circ} \mathrm{C}$ in Biochemical Oxygen Demand (BOD) for 3 days, with a 12-h photoperiod, and two multiplications to achieve greater uniformity. Three days after the second multiplication, Eppendorf tips with an agar disc containing the mycelium were used to inoculate plants of approximately 28 days of age. For inoculation, we eliminated the apex of the main stem of the plant, cutting $2.5 \mathrm{~cm}$ from the node, which remained in contact with the mycelium in the tip. Ten competitive plants per plot were inoculated. Eight days after inoculation, each plant was evaluated for resistance to white mold, by means of a diagrammatic scale as proposed by Terán et al. (2006). The mean of the plot was used for data analyses.

\section{Indirect evaluation of progenies through reaction to oxalic acid}

We evaluated $177 \mathrm{~F}_{2: 4}$ progenies and the parents Jalo and Small White. Seeds $(\mathrm{N}=60)$ of each of the 179 genotypes were sown in Styrofoam trays containing Plantmax ${ }^{\circledR}$ commercial substrate to obtain bean plants. After the emergence of the second trifoliate leaf, about 20 days after sowing, 30 of the most uniform plants were selected.

Due to limited space in the laboratory, nine trials were conducted. In addition to the 179 genotypes, we used two common controls in each experiment, the susceptible cultivar Corujinha and the parent Jalo. The experimental design was completely randomized with three replicates, and each plot contained 10 plants.

Selected plants were cut at the stem base, in the afternoon, and the lower portion of the stem $(2 \mathrm{~cm})$ was immersed in $20 \mathrm{mM}$ oxalic acid solution and kept in a plastic container for $20 \mathrm{~h}$ overnight under controlled temperature $\left(22^{\circ}-24^{\circ} \mathrm{C}\right)$. The solution was adjusted to $\mathrm{pH}$ 4 with $\mathrm{NaOH}$. In all trials, a control (treatment without oxalic acid) was used, consisting of a flask with distilled water $(\mathrm{pH} 4)$ in which some bean plants were immersed. The purpose of this control was to verify if plant wilting was related to the action of oxalic acid.

The reaction of each plant was determined by the descriptive key proposed by Kolkman and Kelly (2000), and the mean of the plot was used for data analysis.

\section{QTL identification}

\section{Moving away from the marker under Bayesian analysis}

Given the low density of polymorphic markers obtained, it was not possible to construct linkage groups consistent with the bean consensus map and, within these maps, the distribution of markers did not permit construction at regular intervals due to the distance of

Genetics and Molecular Research 15 (3): gmr.15038724 
some SSRs in the linkage group. To mitigate this and to exclude marker bias in the search for QTLs, we chose to map multiple QTLs non-biased for marks. This technique was presented by Pamplona et al. (2015), being an adaptation of the multiple-marker analysis described by $\mathrm{Xu}(2003)$ and multiple-QTL analysis described by Wang et al. (2005). It allows the QTL to assume variable positions within a range (distance) set by the frequency of recombination between the QTL and the marker instead of fixed intervals between two flanking marks, such as in the mapping of multiple-QTL mapping, adopting a more restricted search. In this study, we used the recombination frequency range of 0 to 0.25 , adopting a pivotal QTL search in the mark, within this range. Thus, it is assumed that the mark is linked to the QTL with a maximum value of 0.25 , and it then moves within the chosen interval, moving away or approaching the mark that is used as a pivot. Therefore, the likely QTL may be located at any position within this range.

To obtain the corrected mean values, we used the average score obtained in each plot from 10 plants for both experiments. In the straw test evaluation, the averages were corrected based on the recovery of interblock information. The averages obtained in the oxalic acid experiments were subjected to individual and pooled analyses using SAS: PROC MIXED (2000).

For the identification of QTLs, the observed variables are the phenotypic data corrected from averages of progenies $y=\left\{y_{i}\right\}, i=1, \ldots n$ and the genotypes of markers, while unobserved variables are the average $\mathrm{b}_{0}$, the genotypes of QTLs $\left(x_{i j}\right.$ and $\left.w_{i j}\right)$, their effects $\left(a_{j}\right.$ and $\left.d_{j}\right)$, the residual, additive, and dominant variances $\left(\sigma_{0}^{2}, \sigma_{\text {aj }}^{2}\right.$ and $\left.\sigma_{\text {dj }}^{2}\right)$, and the positions of the QTLs, $\lambda$ $=\left\{\lambda_{j}\right\}, j=1, \ldots, p$.

The adopted model is as follows:

$$
y_{i}=b_{o}+\sum_{j=1}^{p} x_{i j} a_{j}+\sum_{j=1}^{p} w_{i j} d_{j}+e_{j}
$$

in which $b_{0}$ is the average of the studied population, $p$ is the total assumed number of QTLs (in this case, the number of markers, assuming that each marker is linked to one QTL), $a_{j}$ and $d_{j}$ are the additive and dominance effects, respectively. The residue $e_{j}$ is assumed to have a normal distribution $N\left(0, \sigma_{o}^{2}\right)$. The variables $x_{i j}$ and $w_{i j}$ are obtained from the metrics $\mathrm{F}_{2}$, as follows (Yang, 2004; Zeng et al., 2005):

$$
x_{i j}=\left\{\begin{array}{l}
1, \text { for AA; } \\
0, \text { for Aa; } \\
-1, \text { for aa. }
\end{array} \text { and } w_{i j}=\left\{\begin{array}{l}
-1 / 2, \text { for AA; } \\
1 / 2, \text { for Aa; } \\
-1 / 2, \text { for aa }
\end{array}\right.\right.
$$

However, $x_{i j}$ and $w_{i j}$ genotypes are not observed, but may be inferred based on the information of marker $j$ and the $\lambda_{\mathrm{j}}$ position of QTLs.

The likelihood function, a priori and a posteriori distributions, and the steps of the Markov Chain Monte Carlo (MCMC) algorithm for sampling of the complete conditional distributions, used in this study were described by Pamplona et al. (2015).

As many ranges do not contain QTLs, they are penalized and shrunk to zero by shrinkage Bayesian methodology. For this, we used a similar formulation to Bayes B, presented by Meuwissen et al. (2001), Gianola et al. (2009), and Gianola (2013). Thus, the hierarchical model can be described as:

$$
y_{i} \mid \beta, \sigma_{0}^{2} \sim N\left(b_{0}+\sum_{j=1}^{p} x_{i j} a_{j}+\sum_{j=1}^{p} w_{i j} d_{j}, \mathrm{I} \sigma_{0}^{2}\right), i=1, \ldots, n ; j=1, \ldots, p \quad \text { (Equation 4) }
$$

Genetics and Molecular Research 15 (3): gmr.15038724 


$$
\begin{aligned}
& a_{j} \sim \sigma_{a_{j}}^{2}, \pi \sim I I D\left\{\begin{array}{c}
N(0, \Delta) \text { with probability } \pi \\
N\left(0, \sigma_{a_{j}}^{2}\right) \text { with probability } 1-\pi
\end{array}, j=1, \ldots, p \quad\right. \text { (Equation 5) } \\
& d_{j} \sim \sigma_{d_{j}}^{2}, \pi \sim I I D\left\{\begin{array}{c}
N(0, \Delta) \text { with probability } \pi \\
N\left(0, \sigma_{d_{j}}^{2}\right) \text { with probability } 1-\pi
\end{array}, j=1, \ldots, p \quad\right. \text { (Equation 6) }
\end{aligned}
$$

The priori is a mixture of a mass of points at 0 (zero) with a Gaussian distribution, where $\Delta$ $=10^{-8}$, and $\pi$ and $1-\pi$ are the mixture probabilities, respectively, where $\pi$ is assumed to be known and arbitrarily specified (herein assumed $\pi=0.95$ ).

For sampling of $\lambda$ positions, the distribution does not present a closed function and, therefore, we used Metropolis-Hastings sampling. Using the priors presented herein, we obtained a model similar to that shown by Pamplona et al. (2015), adapted to Bayes B as described by Meuwissen et al. (2001). The conditional posteriors obtained in this model are similar to those obtained by Pamplona et al. (2015), except that instead of the marker shrinkage factor $\frac{\sigma_{0}^{2}}{\sigma_{a_{j}}^{2}}$ for the additive effect and $\frac{\sigma_{0}^{2}}{\sigma_{a_{j}}^{2}}$ for the dominance effect, the shrinkage factors were $\frac{\sigma_{0}^{2}}{\eta}=\frac{\sigma_{0}^{2}}{\pi \sigma_{a, j}^{2}+(1-\pi) \Delta}$ and $\frac{\sigma_{0}^{2}}{\varphi}=\frac{\sigma_{0}^{2}}{\pi \sigma_{d, j}^{2}+(1-\pi) \Delta}$, respectively, where $\pi:\{1,0\}$ and $p(\pi) \sim \operatorname{Bernoulli}(\pi)$.

$$
p\left(\pi_{j}=1 \mid \ldots\right) \sim \frac{\pi N\left(a_{j} \mid 0, \sigma_{a_{j}}^{2}\right)}{\pi N\left(a_{j} \mid 0, \sigma_{a_{j}}^{2}\right)+(1-\pi) N\left(a_{j} \mid 0, \Delta\right)}
$$

(Equation 7)

\section{Post-MCMC analysis}

The profile of the QTL intensity was represented by Yang and Xu (2007) as a function of position $f(\lambda)$. However, this is not sufficiently informative for inference about the QTL in shrinkage Bayesian analysis. Based on this, Yang and Xu (2007) proposed to describe the effects of QTLs according to their quadratic and weighted forms by the intensity of the position:

$$
g(\lambda)=W(\lambda) f(\lambda)
$$

(Equation 8)

in which $W(\lambda)=b^{\prime} V_{b}^{-1} b+d^{\prime} V_{d}^{-1} d, b$ and $d$ are the vectors of additive and dominant effects, respectively, of QTLs, $V_{b}^{-1}$ and $V_{d}^{-1}$ are the inverses of the variances of the effects of QTLs given by $\left(\sum_{i=1}^{n} w_{i j}^{2}+\frac{\sigma_{0}^{2}}{\varphi}\right)^{-1} \sigma_{0}^{2}$, corresponding to the inverse of the information matrix of additive effect and $\left(\sum_{i=1}^{n} w_{i j}^{2}+\frac{\sigma_{0}^{2}}{\varphi}\right)^{-1} \sigma_{0}^{2}$, which corresponds to the inverse of the information matrix of dominant effect.

This test is equivalent to a Wald test $(W(\lambda))$ and follows an approximate chi-square distribution with two degrees of freedom (Yang and $\mathrm{Xu}, 2007$ ). In this study, the marks considered significant for this population were those with value of (8) greater than $\chi_{(0.95 ; 2)}^{2}=5.99$.

After identifying the likely QTLs, the distances between marks and QTLs were calculated in centimorgans, by using the Kosambi function, which assumes interference in recombination events (Kosambi, 1944). Therefore, the formula used was

Genetics and Molecular Research 15 (3): gmr.15038724 


$$
x=0.25 \cdot \operatorname{In}\left(\frac{1+2 r}{1-2 r}\right)
$$

(Equation 9)

in which $r$ is the recombination frequency.

As the search for QTLs may vary in the range of $r=0.25$, the most probable position of a mark at a certain recombination distance is the mode in the range of 0 to 0.25 . Thus, if the distribution of $\lambda$ is uniform within that range, it is likely to represent no QTL. Alternatively, the intervals can be separated into groups of $1 \mathrm{cM}$ to assess the relative frequency of "hits" in each group. The value of the Wald test can be weighted by the relative frequency (RF) in order to avoid that regions little visited and with high Wald may overlap on regions much visited and with low Wald. This is possible since the change in position in the Metropolis-Hasting algorithm is based on probability $p(y \mid \lambda)$.

The relative frequency results multiplied by Wald (RF x Wald), heritability, and recombination frequencies are presented in graphs. The first graph shown for each evaluation method (oxalic acid and straw test) shows the RF x Wald values of 70 marks used in this study.

In the second graph for each evaluation method, the marks are considered significant (RF x Wald > 5.99) where heritability values were higher than 50\%, and other marks had their heritability values represented by zero, to more clearly visualize the results. The third graph for each evaluation method shows the recombination frequency values of the selected marks in the second graph, and the recombination frequency values of other marks are represented by zero for clear visualization of the final markers selected.

\section{RESULTS}

\section{Segregation of markers in progenies}

Among the 600 pairs of primers tested, 70 were identified as polymorphic in the parents. According to Blair et al. (2006a), common bean possesses no large molecular polymorphisms, even when highly contrasting parents are used. Of the 70 polymorphic primers found, 35 have a known position on 10 of the 11 chromosomes of the species. The positions of the other 35 primers in the genome are not known (BIC, 2007).

Mendelian segregation analysis revealed that 14 of the 70 loci segregated as expected (ATA244, BM139, BM200, BM212, BMD33, BMD36, M75856, PVBR67, PVBR93, PVESTBR_71, PVESTBR_73, PVESTBR_204, PVESTBR_279, and PVM03). Thus, 57 SSR loci $(\overline{81.42 \%})$ showed segregation distortion $(\mathrm{P}<0.05)$.

\section{Identification of QTLs by oxalic acid and straw test}

Before describing the results, it is important to report that the first attempt to identify QTLs involved use of the composite interval mapping method (Zeng, 1993). However, the results were not feasible, since the map (and consequently, the linkage groups) was distorted from the results found in the literature. It should be noted that in situations where the genome has few markers, the constructed genetic map becomes inaccurate and impractical, since it cannot reconstruct the linkage groups or construct linkage maps that are distorted from the physical map. In addition, the presence of a high percentage of loci with segregation distortion

Genetics and Molecular Research 15 (3): gmr.15038724 
may have affected the mapping in this study. In situations such as this, and even with the use of few polymorphic marks, it is necessary to use a method to search QTLs along the genome that does not require map construction. Therefore, we used the adapted technique "moving away from the marker" under Bayesian analysis, since this technique does not require a linkage map to identify QTLs.

Among the 70 markers tested, 62 identified significant QTLs for the expression of white mold resistance through the reaction to oxalic acid. Significance was tested by the Wald test, and by considering the RF of each. Thus, all QTLs with RF x Wald $>5.99$ were considered to be significant. These marks are shown in Figure 1. Markers are represented on the abscissas, and the RF x Wald values are represented on the ordinates. The red line is the limit of 5.99 for identification of QTLs, and markers with peaks represented above this line are considered significant.

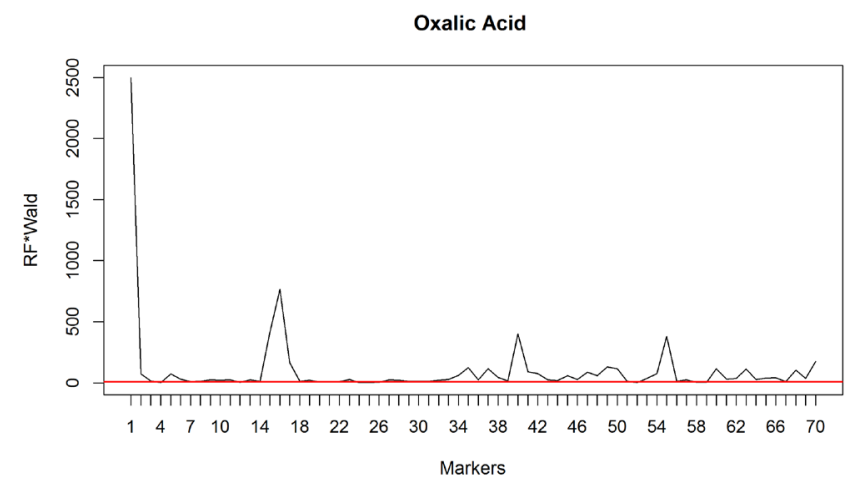

Figure 1. Identification of quantitative trait loci (QTLs) for resistance to white mold by the oxalic acid method with simple sequence repeat (SSR) markers through the relative frequency (RF) $\mathrm{x}$ Wald test, by using the moving away method from the marker under Bayesian analysis.

Among the 62 significant markers, only 10 had heritability higher than 50\% (Figure 2). The red line in Figure 2 represents the limit of 50\% heritability and QTLs considered significant must have peaks above this line to be selected.

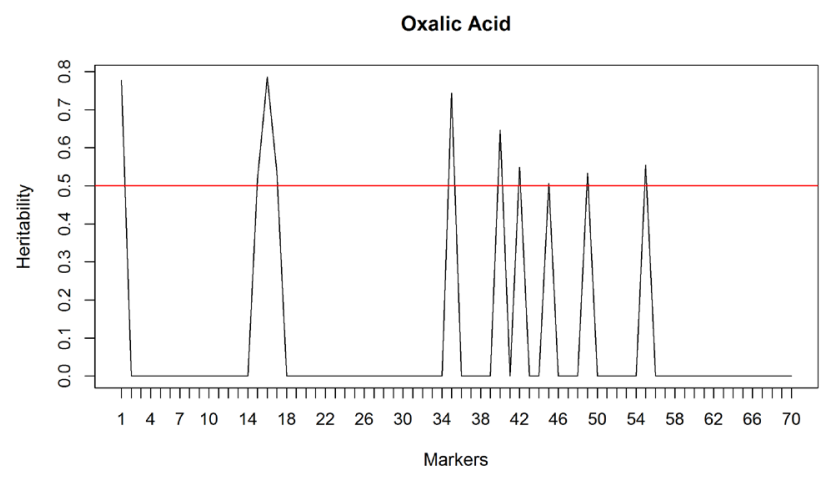

Figure 2. Significant QTLs for resistance to white mold by the oxalic acid method, through SSR markers, with heritability greater than $50 \%$ by using the moving away method from the marker under Bayesian analysis.

Genetics and Molecular Research 15 (3): gmr.15038724 
Importantly, markers with heritability lower than $50 \%$ are represented by zero. This was performed for better visualization of the markers selected in this step.

Heritability measures the reliability of MAS, since it determines the percentage of genetic variation that is inherited. For this type of analysis, no pattern in the literature has defined heritability ranges that can be considered low, medium, or high. Therefore, herein, it was agreed that a minimum of $50 \%$ heritability would be considered acceptable for the use of certain markers in assisted selection. Nevertheless, the higher the value of heritability, the more useful and reliable the marker.

To accept that QTL and marker are linked, we assumed that they must be at a maximum distance of $10 \mathrm{cM}$ (considerable limit to nonoccurrence permutations). In Figure 3, the red line represents the 10-cM recombination frequency, and the markers linked to QTLs, which are considered significant, must have a recombination frequency below this line. Thus, of the 10 significant markers with heritabilities greater than 50\%, seven are linked to QTLs (X57211, BM183, PVBR189, BM189, BM160, PVBR35, and SSR-IAC143) (Figure 3).

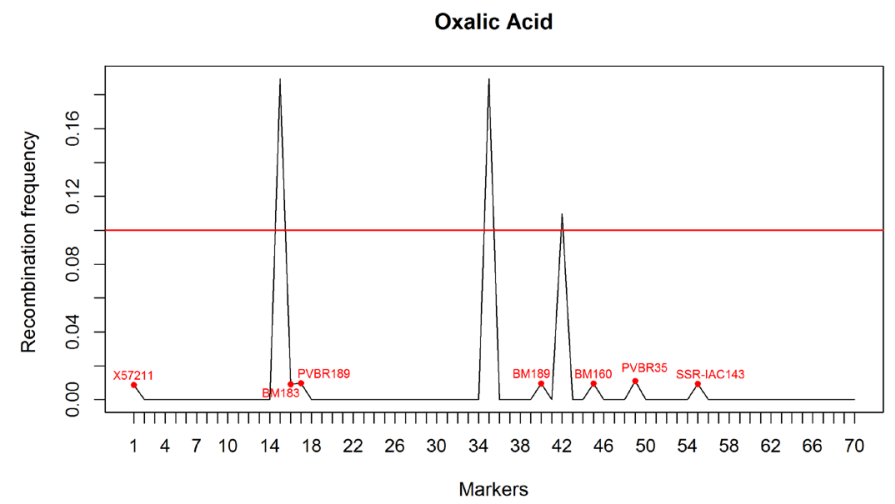

Figure 3. Recombination frequencies between 10 markers and significant QTLs for resistance to white mold by the oxalic acid method, through SSR markers, by using the moving away method from the marker under Bayesian analysis. Red points represent the selected markers.

Of note, markers whose recombination frequencies are represented by zero in Figure 3 did not necessarily present true low values; however, for convenience, they were not represented in these graphs. Such representations were adopted to provide a better view of the peaks of the 10 markers previously selected.

The negative signs of the additive effects indicate that alleles of the resistant line, which has the lowest average, decrease the trait value. As the phenotypic analysis was performed by a grading scale, whereby the lowest score indicates the greatest resistance, the reduction in the trait value leads to disease resistance. Thus, the negative additive effects for QTLs related to the trait indicate that the alleles from the Jalo parental line, which was characterized as resistant, influence this trait, reducing the disease. This is extremely important in plant breeding as only additive effects are transmitted by descent (Juliatti et al., 2013).

Negative dominance effects indicate that heterozygous individuals had lower average values for these traits than the homozygotes, and contribute to greater resistance. As observed for heritability, there is no pattern to define the effect as low, medium, or high. The lower the value (considering the negative effects as favorable), the greater the contribution to disease reduction.

Genetics and Molecular Research 15 (3): gmr.15038724 
For the straw test method, among the 70 markers used, 54 identified significant QTLs, that is, reached RF x Wald > 5.99 (Figure 4).

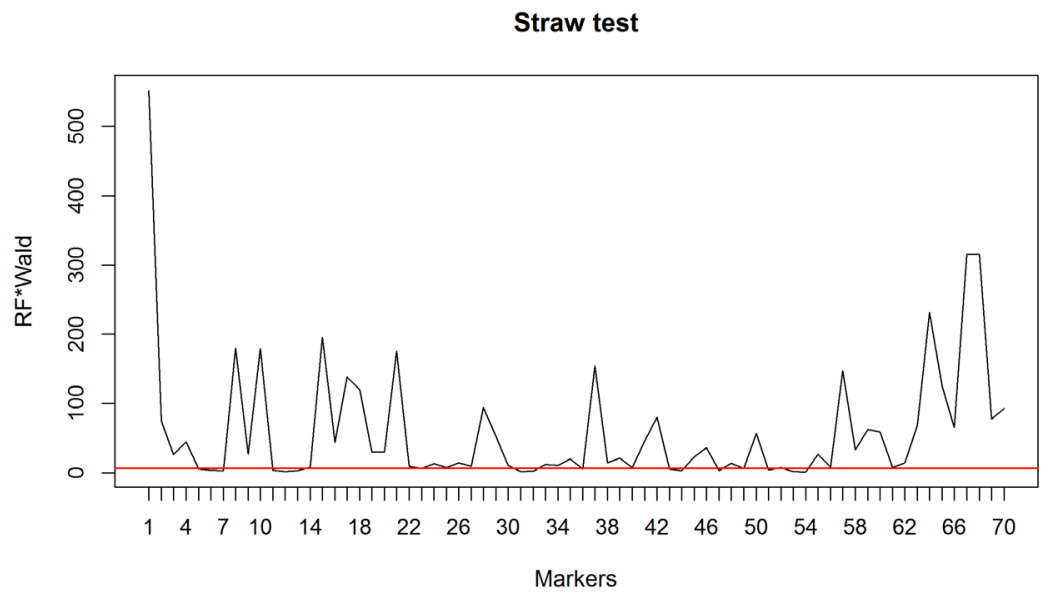

Figure 4. Identification of QTLs for resistance to white mold by the straw test method with SSR markers through the RF x Wald test, by using the moving away method from the marker under Bayesian analysis.

Among these 54 markers, six had heritability of at least 50\%: X57211, PVBR144, PVBR189, X60000, GATS91, and BM197 (Figure 5).

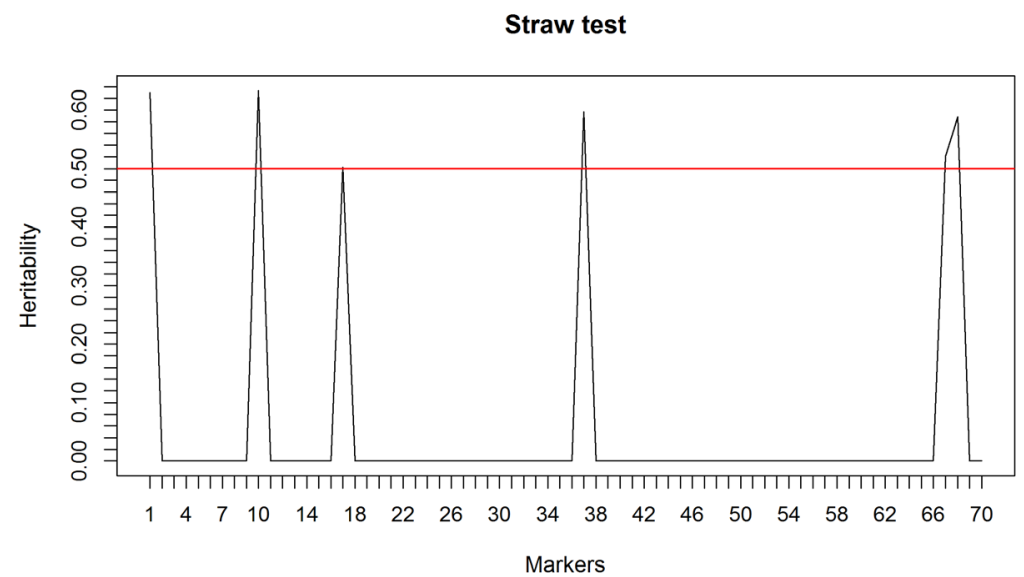

Figure 5. Significant QTLs for resistance to white mold by the straw test method, through SSR markers, with heritability greater than $50 \%$, by using the moving away method from the marker under Bayesian analysis.

Figure 6 shows that all six markers identified as significant in the previous step are located up to $10 \mathrm{cM}$ from a QTL.

Genetics and Molecular Research 15 (3): gmr.15038724 


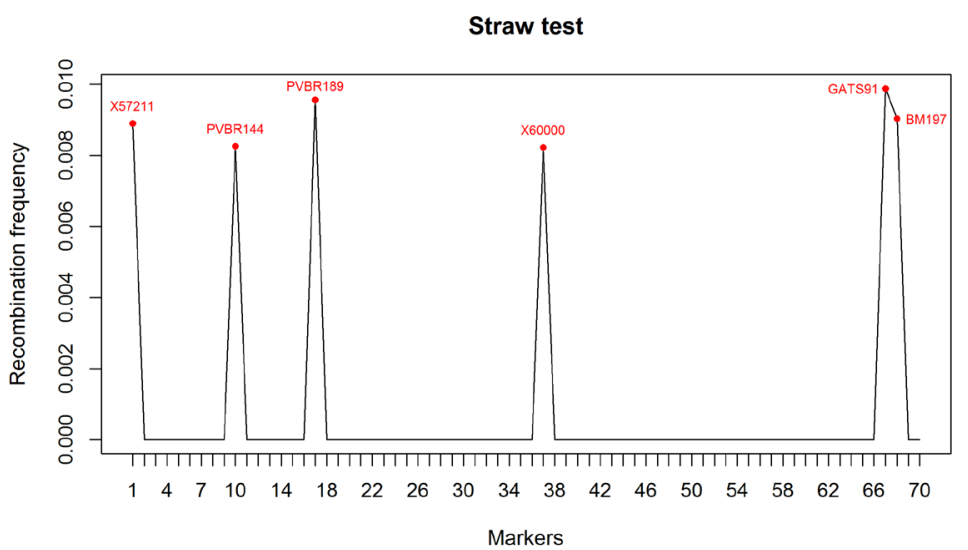

Figure 6. Recombination frequencies between the six markers and significant QTLs for resistance to white mold by the straw test, through SSR markers, by using the moving away method from the marker under Bayesian analysis. Red points represent the selected markers.

Table 1 shows that there was a low coincidence of markers identifying QTLs between the two methods (oxalic acid and straw test). Among the 70 markers used, only two markers with heritability greater than $50 \%$ simultaneously identified QTLs by straw test and oxalic acid, with variation in the effects of reduced or increased resistance. The QTL found in both tests was almost at the same distance, which could indicate a pleiotropic effect. However, given the distance between the mark and the identified QTL, these two marks would not be very useful in plant breeding. Given the distance between the mark and the QTL found, these two marks would be very useful in common bean breeding, especially PVBR189, for increasing the resistance.

Table 1. Consistencies between markers near QTLs determined by oxalic acid analysis $\left(\mathrm{F}_{2: 4}\right.$ progenies $)$ and the straw test $\left(\mathrm{F}_{2: 3}\right.$ progenies) in common bean.

\begin{tabular}{|c|c|c|c|c|c|}
\hline Marker & $\mathrm{D}^{\mathrm{a}}$ & E.ad ${ }^{b}$ & E. Dom ${ }^{c}$ & $h^{2}(\%)^{d}$ & RF $\times$ Wald \\
\hline \multicolumn{6}{|l|}{ Oxalic acid } \\
\hline X57211 & 0.862 & + & + & 77.765 & 2500.330 \\
\hline PVBR189 & 0.983 & - & - & 53.671 & 166.489 \\
\hline \multicolumn{6}{|l|}{ Straw test } \\
\hline X57211 & 0.890 & + & + & 63.004 & 551.893 \\
\hline PVBR189 & 0.956 & - & - & 50.284 & 138.647 \\
\hline
\end{tabular}

a Distance in centimorgan (cM) between the marker and the QTL; ${ }^{\mathrm{b}}$ Quantitative trait loci (QTL) additive effect;

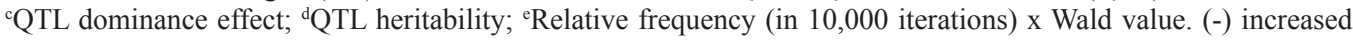
resistance; $(+)$ reduced resistance.

\section{DISCUSSION}

Markers with segregation distortion in studies on common bean have been detected in the literature. Blair et al. (2003) found 23 and 9\% segregation distortion in SSR loci in two different populations. Galeano et al. (2009) and Garcia et al. (2011) detected 19 and 28.9\% of loci with significant segregation distortion, respectively.

Genetics and Molecular Research 15 (3): gmr.15038724 
According to Tanksley et al. (1992), Mendelian segregation distortion along the genome may be caused by chromosomal rearrangements and post-zygotic or zygotic gametic selection. The sampling effect and genotyping errors may also contribute. It is likely that some of those factors contributed to the high percentage of loci with distorted segregation in the present study.

Although the exact cause of segregation distortion is unknown, some studies have shown the importance of these loci in QTL identification from genetic maps. Xu (2008) suggested a theory for the emergence of loci with segregation deviation and stated that QTL detection power can be subtly reduced when the analysis disregards deviated loci. The author also noted that if the map is well saturated, and such loci are randomly distributed in the genome, the losses are insignificant. They also argued that loci with segregation deviation are used to obtain a genetic map and for QTL identification, because when the marker is closely linked to the QTL, the identification made by the marker is not affected.

Some of the markers associated with white mold have been described in other studies in relation to the disease, whether from evaluation of the occurrence in the field, by inoculation through the straw test, or by the reaction to oxalic acid.

Markers BMD33 (GL11) and BM157 (GL1) were mapped by Mkwaila (2013): BM157 was associated with QTL WM1.3 ${ }^{\mathrm{AP} 630}$, which explains $19.9 \%$ of the variation in white mold resistance as assessed by the incidence in the field; and BMD33 was associated with QTL WM11.1 ${ }^{\mathrm{TL}}$, explaining 15.4\% variation in the expression of resistance to white mold by straw test. In the present study, despite markers BM157 (straw test) and BMD33 (oxalic acid) presenting significant QTLs for resistance to white mold, both showed low heritability (16.69 and $15.12 \%$, respectively).

Ender and Kelly (2005), Maxwell et al. (2007), and Mkwaila (2013) mapped the marker BM143 in GL2 near the QTL designated WM2.3 $3^{\mathrm{BR}, \mathrm{GC}, \mathrm{TW}}$. However, in the present study, the marker BM143 only identified significant QTL by the oxalic acid method, which has low heritability $(24.72 \%)$, despite its negative dominance effect.

Haggard (2007) showed that GL2 are contained in the marker BM139, which is strongly associated with resistance to white mold in the field, and GATS91, which is associated with resistance both in the field and in the straw test. In the present study, BM139 identified QTL with low heritability. GATS91 also identified QTL through both approaches, and was more promising for MAS in the straw test evaluation because it acts to reduce disease (additive and dominance effects of -0.284 and -0.011 , respectively) with $52.06 \%$ heritability.

In the map produced by Haggard (2007), GL3 has the marker BM172 associated with resistance to white mold in the field and in the straw test. This marker identified QTLs by both methodologies, with effects in disease reduction; however, it showed low heritability (39.75 and $27.73 \%$ for the oxalic acid and straw test methods, respectively).

Marker BM187 was mapped in GL6, identifying the QTL WM6.1 ${ }^{\mathrm{B} 60}$, R31 from populations RIL Benton/NY6020-4 (Miklas et al., 2003) and RIL Raven/19365-31 (Soule et al., 2011). This marker flanks QTLs for days-to-flowering, plant height, plant width, number of seeds per plant, and the $\mathrm{V}$ gene, which is associated with flower color (Blair et al., 2006b). Haggard (2007) identified this marker associated with resistance to white mold by straw test in GL6. By using both the straw test and reaction to oxalic acid, Lara et al. $(2014,2015)$ used the mapping population obtained from a cross between CNFC 9506 and RP-2 and identified the markers BM184 and BM187 associated with the most significant QTL, with high heritability. However, these markers contribute to reduce resistance and are distant from each other at 43.84 cM by the oxalic acid method. In the present study, although BM187 was linked to a QTL (9.10

Genetics and Molecular Research 15 (3): gmr.15038724 
$\mathrm{cM})$ and acted to reduce the disease, it presented low heritability (30.86\%) by oxalic acid.

Marker ME1 was mapped by Galeano et al. (2009) and Blair et al. (2010) in GL9. In a study developed by Lara et al. $(2014,2015)$, ME1 was found to be located 0.82 and 0.29 cM from a QTL with 71.37 and $0.55 \%$ heritability by the straw test and oxalic acid evaluation methods, respectively. This QTL acts to increase resistance to white mold. In the present study, ME1 also identified QTLs by both evaluation methods; however, the heritability obtained was low, being $17.31 \%$ when using oxalic acid and $27.70 \%$ when using the straw test.

Marker BM183 was mapped in GL7 by Gaitán-Solís et al. (2002). In the present study, this marker was efficient at identifying a QTL by both methodologies, which works by reducing the disease, because the additive and dominance effects are negative. This marker would be more promising in selection by the oxalic acid method as a heritability of $78.59 \%$ was presented when using this method.

Other QTLs related to white mold resistance have been reported in the literature; however, the markers that identified them were not used in the present study. In addition, some markers were associated with resistance to white mold in this study but were not supported by evidence from the literature. It is important to highlight them, since different results were found in other studies and because the resistant parent (Jalo) is of Andean origin and there have been no reports on its use in other studies.

Using the oxalic acid method, further notable markers were BM189 and SSR-IAC143, because they had higher RF x Wald values and effects at reducing white mold.

Using the straw test method, markers X60000 and BM197 stood out with heritability above $50 \%$ and effects on increasing resistance. The results of the present study indicate that marker BM197 is $0.903 \mathrm{cM}$ from a QTL; X6000 is $0.822 \mathrm{cM}$ from the identified QTL. These markers showed the smallest additive effect (BM197) and the smallest dominance effect (X60000), i.e., greater power of white mold reduction in the population and are considered as promising in MAS.

Considering the two evaluation methods, Table 1 shows the coincidence, mainly, for the QTL identified by marker PVBR189, which acts by increasing resistance. This marker is therefore important for the simultaneous selection of resistance by both the oxalic acid and straw test methods. The marker X57211 acts by reducing resistance to disease, despite identifying significant QTL.

A low number of coincident markers between the two methodologies was expected, given the low and non-significant correlation (Spearman correlation of 2.06\%) between the averages of the progenies by both methods. In addition, the coincidence between the two QTLs discussed indicates the possibility of identifying the same QTL for both resistance mechanisms. However, confirmation of this hypothesis is only possible by mapping these QTLs and by analyzing the population with the highest number of markers; therefore, this was inconclusive in the present study.

With the lack of correlation between phenotypic evaluations by the oxalic acid test and the straw test, these results confirm that there are multiple genes/QTL controlling the physiological resistance to white mold, that is, some genotypes having higher tolerance to oxalic acid, while others having additional resistance identified by straw test. There have been some suggestions that oxalic acid released by S. sclerotiorum contributes to the initial aggression of the fungus to the host, besides acting in the subsequent stages of defense (Cessna et al., 2000).

In conclusion, markers GATS91, BM197, and X60000 stood out with high heritability and with the greatest effects in reducing the disease by straw test. Thus, they are promising for MAS.

Genetics and Molecular Research 15 (3): gmr.15038724 
Markers BM183, BM189, and SSR-IAC143 are associated with QTLs identified by oxalic acid, contributing to the greatest effects in increasing resistance to white mold, with high heritability.

Considering the simultaneous use of the oxalic acid and straw test methods, the most prominent marker was PVBR189. However, all are relevant to MAS and may contribute to increase disease resistance.

\section{Conflicts of interest}

The authors declare no conflict of interest.

\section{ACKNOWLEDGMENTS}

The authors thank Conselho Nacional de Desenvolvimento Científico e Tecnológico (CNPq) and Coordenação de Aperfeiçoamento de Pessoal de Nível Superior (CAPES).

\section{REFERENCES}

BIC (2007). Bean Improvement Cooperative. Genetics: bean SSR primers. Washington. Available at [http://www.css. msu.edu/bic/PDF/Bean_SSR_Primers_2007.pdf]. Accessed December 20, 2015.

Blair MW, Pedraza F, Buendia HF, Gaitán-Solís E, et al. (2003). Development of a genome-wide anchored microsatellite map for common bean (Phaseolus vulgaris L.). Theor. Appl. Genet. 107: 1362-1374. http://dx.doi.org/10.1007/ $\underline{\text { s00122-003-1398-6 }}$

Blair MW, Iriarte G and Beebe S (2006a). QTL analysis of yield traits in an advanced backcross population derived from a cultivated Andean x wild common bean (Phaseolus vulgaris L.) cross. Theor. Appl. Genet. 112: 1149-1163. http:// dx.doi.org/10.1007/s00122-006-0217-2

Blair MW, Giraldo MC, Buendía HF, Tovar E, et al. (2006b). Microsatellite marker diversity in common bean (Phaseolus vulgaris L.). Theor. Appl. Genet. 113: 100-109. http://dx.doi.org/10.1007/s00122-006-0276-4

Blair MW, Medina JI, Astudillo C, Rengifo J, et al. (2010). QTL for seed iron and zinc concentration and content in a Mesoamerican common bean (Phaseolus vulgaris L.) population. Theor. Appl. Genet. 121: 1059-1070.http://dx.doi. org/10.1007/s00122-010-1371-0

Carneiro FF, Santos JB and Leite ME (2010). Marker-assisted backcrossing using microsatellites and validation of SCAR Phs marker for resistance to white mold in common bean. Electron. J. Biotechnol. 13: 9-10.

Carvalho RSB, Lima IA, Alves FC and Santos JB (2013). Selection of carioca common bean progenies resistant to white mold. Crop Breed. Appl. Biotechnol. 13: 172-177.

Cessna SG, Sears VE, Dickman MB and Low PS (2000). Oxalic acid, a pathogenicity factor for Sclerotinia sclerotiorum, suppresses the oxidative burst of the host plant. Plant Cell 12: 2191-2200. http://dx.doi.org/10.1105/tpc.12.11.2191

Ender M and Kelly JD (2005). Identification of QTL associated with white mold resistance in common bean. Crop Sci. 45: 2482-2490. http://dx.doi.org/10.2135/cropsci2005.0064

Gaitán-Solís E, Duque MC, Edwards KJ and Tohme J (2002). Microsatellite repeats in common bean (Phaseolus vulgaris) isolation, characterization, and cross-species amplification in Phaseolus ssp. Crop Sci. 42: 2128-2136.

Galeano CH, Fernández AC, Gómez M and Blair MW (2009). Single strand conformation polymorphism based SNP and Indel markers for genetic mapping and synteny analysis of common bean (Phaseolus vulgaris L.). BMC Genomics 10: 629-630. http://dx.doi.org/10.1186/1471-2164-10-629

Garcia RA, Rangel PN, Brondani C, Martins WS, et al. (2011). The characterization of a new set of EST-derived simple sequence repeat (SSR) markers as a resource for the genetic analysis of Phaseolus vulgaris. BMC Genet. 12: 41. http://dx.doi.org/10.1186/1471-2156-12-41

Gianola D (2013). Priors in whole-genome regression: the bayesian alphabet returns. Genetics 194: 573-596. http://dx.doi. org/10.1534/genetics.113.151753

Gianola D, de los Campos G, Hill WG, Manfredi E, et al. (2009). Additive genetic variability and the Bayesian alphabet. Genetics 183: 347-363. http://dx.doi.org/10.1534/genetics.109.103952

Haggard JE (2007). Characterization of Physiological Resistance to White Mold and Search for Molecular Markers Linked to Resistance via Advanced Backcross QTL Analysis in an Interspecific Cross between Phaseolus coccineus and $P$. vulgaris. Master's thesis. Oregon State University.

Genetics and Molecular Research 15 (3): gmr.15038724 
Juliatti FC, Pedrosa MG, Juliatti BCM, Beloti IF, et al. (2013). Identificação de QTLs associados à resistência parcial à mancha branca do milho. Biosci. J. 29: 1163-1178.

Kolkman JM and Kelly JD (2000). An indirect test using oxalate to determine physiological resistance to white mold in common bean. Crop Sci. 40: 281-285. http://dx.doi.org/10.2135/cropsci2000.401281x

Kosambi DD (1944). The estimation of map distances from recombination values. Ann. Eugen. 12: 172-175. http://dx.doi. org $/ 10.1111 / \mathrm{j} .1469-1809.1943 . t b 02321 . x$

Lara LAC, Dos Santos JB, Veloso JS, Balestre M, et al. (2014). Identification of QTLs for resistance to Sclerotinia sclerotiorum in carioca common bean by the moving away method. ISRN Mol. Biol. 2014: 828102 http://dx.doi. org/10.1155/2014/828102.

Lara LAC, Santos JB, Balestre M, Lima IA, et al. (2015). Identification of QTLs of resistance to white mold in common bean from multiple markers by using Bayesian analysis. Genet. Mol. Res. 14: 1124-1135.http://dx.doi.org/10.4238/2015. February.6.16

Maxwell JJ, Brick MA, Byrne PF, Schwartz HF, et al. (2007). Quantitative trait loci linked to white mold resistance in common bean. Crop Sci. 47: 2285-2296. http://dx.doi.org/10.2135/cropsci2007.01.0022

Meuwissen THE, Hayes BJ and Goddard ME (2001). Prediction of total genetic value using genome-wide dense marker maps. Genetics 157: 1819-1829.

Miklas PN, Delorme R and Riley R (2003). Identification of QTL conditioning resistance to white mold in snap bean. $J$. Am. Soc. Hortic. Sci. 128: 564-570.

Mkwaila WE (2013). Quantitative Trait Loci Analysis of Resistance to White Mold (Sclerotinia sclerotiorum) in Common Bean (Phaseolus vulgaris). Doctoral dissertation. Michigan State University.

Pamplona AKA, Balestre M, Lara LAC, Santos JB, et al. (2015). A new method of QTL identification for undersaturated maps. Genet. Mol. Res. 14: 11462-11479. http://dx.doi.org/10.4238/2015.September.25.13

Pereira HS, Santos JD, Abreu ADF and Couto KR (2007). Informações fenotípicas e marcadores microssatélites de QTL na escolha de populações segregantes de feijoeiro. Pesqui. Agropecu. Bras. 42: 707-713. http://dx.doi.org/10.1590/ $\underline{\mathrm{S} 0100-204 X 2007000500014}$

Silva PH, Santos JB, Lima IA, Lara LA, et al. (2014). Reaction of common bean lines and aggressiveness of Sclerotinia sclerotiorum isolates. Genet. Mol. Res. 13: 9138-9151.http://dx.doi.org/10.4238/2014.November.7.11

Singh SP and Téran H (2008). Evolution of screening methods for detection of physiological resistance to white mold in common bean. Annu. Rep. Bean Improv. Coop. 51: 40-41.

Singh SP and Schwartz HF (2010). Breeding common bean for resistance to diseases: A review. Crop Sci. 50: 2199-2223. http://dx.doi.org/10.2135/cropsci2009.03.0163

Soule M, Porter L, Medina J, Santana GP, et al. (2011). Comparative QTL Map for white mold resistance in common bean, and characterization of partial resistance in dry bean lines VA19 and I9365-31. Crop Sci. 51: 123-139. http://dx.doi. org/10.2135/cropsci2010.06.0356

Statistical Analysis System (SAS) Institute (2000). SAS/STATS user's guide. Version 8. Cary.

Tanksley SD, Ganal MW, Prince JP, de Vicente MC, et al. (1992). High density molecular linkage maps of the tomato and potato genomes. Genetics 132: 1141-1160.

Terán H, Lema M, Schwartz HF, Duncan R, et al. (2006). Modified petzoldt and dickson scale for white mold rating of Common bean. Annu. Rep. Bean Improv. Coop. 49: 115-116.

Wang H, Zhang YM, Li X, Masinde GL, et al. (2005). Bayesian shrinkage estimation of quantitative trait loci parameters. Genetics 170: 465-480.http://dx.doi.org/10.1534/genetics.104.039354

Xu S (2003). Estimating polygenic effects using markers of the entire genome. Genetics 163: 789-801.

Xu S (2008). Quantitative trait locus mapping can benefit from segregation distortion. Genetics 180: 2201-2208. http:// dx.doi.org/10.1534/genetics.108.090688

Yang R and Xu S (2007). Bayesian shrinkage analysis of quantitative trait Loci for dynamic traits. Genetics 176: 11691185. http://dx.doi.org/10.1534/genetics.106.064279

Yang R-C (2004). Epistasis of quantitative trait loci under different gene action models. Genetics 167: 1493-1505. http:// dx.doi.org/10.1534/genetics.103.020016

Zeng ZB (1993). Theoretical basis for separation of multiple linked gene effects in mapping quantitative trait loci. Proc. Natl. Acad. Sci. USA 90: 10972-10976.http://dx.doi.org/10.1073/pnas.90.23.10972

Zeng ZB, Wang T and Zou W (2005). Modeling quantitative trait Loci and interpretation of models. Genetics 169: 17111725. http://dx.doi.org/10.1534/genetics.104.035857

Genetics and Molecular Research 15 (3): gmr.15038724 